Valentin A. Skvortsov* and Alexei P. Solodov`ं Mechanics and Mathematics Department, Moscow State University, 119899, Russia; e-mail:

vaskvor@nw.math.msu.su

\title{
A VARIATIONAL INTEGRAL FOR BANACH-VALUED FUNCTIONS
}

\begin{abstract}
It is shown that for Banach-space-valued functions the variational Henstock integral is equivalent to the Henstock integral if and only if the range space is of a finite dimension. The same is true for the equivalence of the variational McShane integral and the McShane integral.
\end{abstract}

There are various ways to define the Henstock integral. The original Henstock-Kurzweil definition is based on generalized Riemann sums (the $H$-integral). For real-valued functions this integral is known to be equivalent to the variational Henstock integral (the $V$-integral, see [10]) and to the Denjoy-Perron integral (the $D_{*}$-integral, see [7]). The first of this equivalence is a corollary of the so-called Saks-Henstock Lemma (see Lemma 1 below).

Here we are considering Henstock type integral for Banach-space-valued functions. It was noticed by S. S. Cao in [2] that for such functions SaksHenstock Lemma might fail to be true. Because of that for some spaces the $V$-integral is not equivalent to the $H$-integral. It is natural to ask what is a characterization of those Banach spaces for which such equivalence holds.

We are showing here that for Banach-space-valued functions the $V$-integral is equivalent to the $H$-integral if and only if the range space is of a finite dimension. At the same time for any Banach space the $V$-integral is equivalent to the Denjoy-Bochner integral.

Similar problems are considered for the variational McShane integral.

First we recall some notations and definitions. We denote by $X$ a Banach space with the norm $\|\cdot\|$, by $\mathbb{R}$ the real line, by $[a, b]$ a closed interval on the line, and by $|E|$ Lebesgue measure of a set $E$.

Key Words: Henstock integral, McShane integral, variational integral, Bochner integral, Banach-valued functions

Mathematical Reviews subject classification: 28B05, 26A39, 46G10

Received by the editors April 16, 1998

*Partially supported by the Institute of Mathematics, WSP, Bydgoszcz, Poland

†Supported by RFFI (Grants 96-01-00332 and 96-15-96073) 
Let $\mathcal{I}$ be a collection of all closed intervals that are contained in $[a, b]$. A collection $T$ of pairs $\left(\Delta_{k}, \xi_{k}\right) \in \mathcal{I} \times[a, b], i=1, \ldots, n$, is called a partition of the interval $[a, b]$ if the intervals $\Delta_{i}$ and $\Delta_{j}$ are non-overlapping for $i \neq j$, and $\bigcup_{k=1}^{n} \Delta_{k}=[a, b]$.

Let $\delta:[a, b] \longrightarrow(0, \infty)$ be a positive function defined on $[a, b]$. A partition $T$ of $[a, b]$ is called Henstock $\delta$-fine if every pair $(\Delta, \xi) \in T$ satisfies

$$
\xi \in \Delta \subset(\xi-\delta(\xi), \xi+\delta(\xi)) .
$$

Definition 1. ([6]). A function $f:[a, b] \longrightarrow X$ is called Henstock integrable (H-integrable) on a closed interval $[a, b]$ with integral value $I \in X$ if for every $\varepsilon>0$ there exists a positive function $\delta:[a, b] \longrightarrow(0, \infty)$ such that for every Henstock $\delta$-fine partition $T$ of $[a, b]$

$$
\left\|\sum_{T} f\left(\xi_{k}\right)\left|\Delta_{k}\right|-I\right\|<\varepsilon
$$

We denote $I=(H) \int_{a}^{b} f d t$.

A partition $T$ of $[a, b]$ is called McShane $\delta$-fine ( $\delta$ being a positive function on $[a, b])$ if every pair $(\Delta, \xi) \in T$ satisfies

$$
\Delta \subset(\xi-\delta(\xi), \xi+\delta(\xi)) .
$$

Definition 2. ([9]). A function $f:[a, b] \longrightarrow X$ is called McShane integrable (M-integrable) on a closed interval $[a, b]$ with integral value $I \in X$ if for every $\varepsilon>0$ there exists a positive function $\delta:[a, b] \longrightarrow(0, \infty)$ such that for every McShane $\delta$-fine partition $T$ of $[a, b]$

$$
\left\|\sum_{T} f\left(\xi_{k}\right)\left|\Delta_{k}\right|-I\right\|<\varepsilon
$$

We denote $I=(M) \int_{a}^{b} f d t$.

It is clear that $M$-integrability implies $H$-integrability. Since $H$-integrability and $M$-integrability on $[a, b]$ imply integrability on any subinterval $\Delta \subset[a, b]$, we can define the indefinite $H$-integral and the indefinite $M$-integral by putting $F(\Delta)=(H) \int_{\Delta} f d t\left(F(\Delta)=(M) \int_{\Delta} f d t\right)$.

Let $\Phi: \mathcal{I} \times[a, b] \longrightarrow X$ be an interval- -point function. The Henstock and the McShane variations of $\Phi$ are defined as

$$
\mathrm{V}_{H}(\Phi)=\inf _{\delta} \sup _{T} \sum_{T} \| \Phi\left(\xi_{k}, \Delta_{k} \|\right.
$$


(sup is taken over all Henstock $\delta$-fine partitions $\mathrm{T}$ and inf is taken over all positive functions $\delta$ on $[a, b]$ ), and

$$
\mathrm{V}_{M}(\Phi)=\inf _{\delta} \sup _{T} \sum_{T} \| \Phi\left(\xi_{k}, \Delta_{k} \|\right.
$$

(sup is taken over all McShane $\delta$-fine partitions $T$ and inf is taken over all positive functions $\delta$ on $[a, b])$.

Note that any interval function $\Phi: \mathcal{I} \longrightarrow X$ can be considered as an interval-point function dependent only on the first argument.

The following two definitions of variational integrals are natural extensions of the definitions for the real-valued case (see [10]).

Functions $\Phi_{1}, \Phi_{2}: \mathcal{I} \times[a, b] \longrightarrow X$ are said to be Henstock variationally equivalent if $\mathrm{V}_{H}\left(\Phi_{1}-\Phi_{2}\right)=0$.

Definition 3. A function $f:[a, b] \longrightarrow X$ is called Henstock variationally integrable ( $V$-integrable) on $[a, b]$ if there exists an additive interval function $F: \mathcal{I} \longrightarrow X$ such that the interval-point function $f(t)|\Delta|$ and $F(\Delta)$ are Henstock variationally equivalent, $F(\Delta)$ being the indefinite $V$-integral of $f$.

Functions $\Phi_{1}, \Phi_{2}: \mathcal{I} \times[a, b] \longrightarrow X$ are said to be McShane variationally equivalent if $\mathrm{V}_{M}\left(\Phi_{1}-\Phi_{2}\right)=0$.

Definition 4. A function $f:[a, b] \longrightarrow X$ is called McShane variationally integrable ( $M V$-integrable) on $[a, b]$ if there exists an additive interval function $F: \mathcal{I} \longrightarrow X$ such that the interval-point function $f(t)|\Delta|$ and $F(\Delta)$ are McShane variationally equivalent, $F(\Delta)$ being the indefinite $M V$-integral of $f$.

Definition 5. ([8]). A function $F: \mathcal{I} \longrightarrow X$ is said to be $A C$-function on a set $E \subset[a, b]$ if for every $\varepsilon>0$ there exists $\delta>0$ such that for every collection of non-overlapping closed intervals $\left\{\Delta_{i}\right\}_{i=1}^{n}$ with the end points belonging to $E$ and with $\sum_{i=1}^{n}\left|\Delta_{i}\right|<\delta$, we have $\sum_{i=1}^{n}\left\|F\left(\Delta_{i}\right)\right\|<\varepsilon$.

Definition 6. ([8]). A function $f:[a, b] \longrightarrow X$ is said to be Bochner integrable (B-integrable) on $[a, b]$, if there exists a function $F: \mathcal{I} \longrightarrow X$ that is $A C$ on $[a, b]$ and such that it is differentiable a. e. and $F^{\prime}(t)=f(t)$ a. e. on $[a, b]$, $F(\Delta)$ being the indefinite $B$-integral of $f$.

Definition 7. ([1]). A function $F: \mathcal{I} \longrightarrow X$ is said to be $A C^{*}$-function on a set $E \subset[a, b]$ if for every $\varepsilon>0$ there exists $\delta>0$ such that for every collection of non-overlapping closed intervals $\left\{\Delta_{i}\right\}_{i=1}^{n}$ with one of the end points belonging to $E$ and with $\sum_{i=1}^{n}\left|\Delta_{i}\right|<\delta$, we have $\sum_{i=1}^{n}\left\|F\left(\Delta_{i}\right)\right\|<\varepsilon$.

It is clear that for $E=[a, b]$ the class of $A C^{*}$-functions coincides with the class of $A C$-functions. 
Definition 8. ([1]). A function $F: \mathcal{I} \longrightarrow X$ is said to be $A C G^{*}$-function on a set $E \subset[a, b]$ if $E$ can be represented as a union of a sequence of sets such that $F$ is $A C^{*}$-function on each of them.

Definition 9. ([1]). A function $f:[a, b] \longrightarrow X$ is said to be Denjoy-Bochner integrable $\left(D_{*} B\right.$-integrable) on $[a, b]$, if there exists an $A C G^{*}$-function $F$ : $\mathcal{I} \longrightarrow X$ such that it is differentiable a. e. and $F^{\prime}(t)=f(t)$ a. e. on $[a, b]$, $F(\Delta)$ being the indefinite $D_{*} B$-integral of $f$.

The Definitions 5 and $7-9$ are extensions of the respective definitions for the real-valued case (see [11]).

The following proposition is a direct corollary of the definitions.

Proposition 1. If $f:[a, b] \longrightarrow X$ is $V$-integrable ( $M V$-integrable) on $[a, b]$ then it is also $H$-integrable ( $M$-integrable)on $[a, b]$ and the indefinite integrals coincide.

The following assertion is known as Saks-Henstock Lemma for real-valued functions and is easily extended to the case of vector-valued functions with range spaces being spaces of finite dimensions.

Lemma 1. ([3]). Let $X$ be a Banach space of a finite dimension. If a function $f:[a, b] \longrightarrow X$ is $H$-integrable (M-integrable) with the indefinite integral $F: \mathcal{I} \longrightarrow X$ then for every $\varepsilon>0$ there exists a function $\delta:[a, b] \longrightarrow(0, \infty)$ such that for every Henstock (McShane) $\delta$-fine partition $T$ of $[a, b]$

$$
\sum_{T}\left\|f\left(\xi_{k}\right)\left|\Delta_{k}\right|-F\left(\Delta_{k}\right)\right\|<\varepsilon
$$

S. S. Cao (see [3]) introduced a definition of $H L$-integrability of a function $f:[a, b] \longrightarrow X$ which is a restriction of $H$-integrability by the requirement that the assertion of Lemma 1 is valid for $f$. It is proved in [13] that the $H L$-integral is equivalent to the $D_{*} B$-integral. (The same equivalence was stated in [1], but there was some gap in the proof which was overcome in [13].) Since $V$-integrability of a function $f:[a, b] \longrightarrow X$ is obviously equivalent to the assertion of Saks-Henstock Lemma we get

Theorem 1. Let $X$ be a Banach space. For functions taking values in $X$ the $V$-integral is equivalent to the $D_{*} B$-integral.

Analogous fact for the $M V$-integral is the following one.

Theorem 2. Let $X$ be a Banach space. For functions taking values in $X$ the $M V$-integral is equivalent to the B-integral. 
The proof is the same as above. It is enough to use [4] instead of [13]. Proposition 1 and Theorems 1 and 2 imply

Proposition 2. If a function $f:[a, b] \longrightarrow X$ is $D_{*} B$-integrable (B-integrable) it is also $H$-integrable ( $M$-integrable) and the indefinite integrals coincide.

Now we consider the relation between the $H$-integral (the $M$-integral) and the $V$-integral (the $M V$-integral). Our aim in the rest of the paper is to prove the following theorem.

Theorem 3. Consider functions on $[a, b]$ taking values in a fixed Banach space $X$. Then the $V$-integral (the $M V$-integral) is equivalent to the $H$-integral (the $M$-integral) on this class of functions if and only if $X$ is of a finite dimension.

Proof. The sufficiency follows easily from Lemma 1. The proof of the necessity is based on a geometric idea (see [12]) which in turn follows from the construction by A. Dvoretzky and C. A. Rogers used in [5] to show that in every infinite-dimensional Banach space there exists a series that is unconditionally but not absolutely convergent.

Lemma 2. ([5]). Let $B$ be a body in $\mathbb{R}^{n}$ which is convex and has the origin as a center and let $r$ be an integer with $1 \leq r \leq n$. Then there exist $r$ vectors $A_{1}, A_{2}, \ldots, A_{r} \in \mathbb{R}^{n}$ on the boundary of $B$ such that if $\lambda_{1}, \lambda_{2}, \ldots, \lambda_{r}$ are any $r$ real numbers then

$$
\sum_{i=1}^{r} \lambda_{i} A_{i} \in \lambda B, \text { where } \lambda^{2}=\left(2+\frac{r(r-1)}{n}\right) \sum_{i=1}^{r} \lambda_{i}^{2} \text {. }
$$

$(\lambda B$ is the set $\{\lambda x, x \in B\}$.

Lemma 3. Let $X$ be a Banach space of the infinite dimension. Then for every natural number $r$ there exist unit vectors $x_{1}, x_{2}, \ldots, x_{r} \in X$ such that for every numbers $\theta_{1}, \theta_{2}, \ldots, \theta_{r}$ with $\left|\theta_{i}\right| \leq 1,1 \leq i \leq r$,

$$
\left\|\sum_{i=1}^{r} \theta_{i} x_{i}\right\|^{2} \leq 3 r
$$

Proof. Since $X$ is of the infinite dimension, for any $n$ there exist linear independent vectors $z_{1}, z_{2}, \ldots, z_{n} \in X$. Take $n=r(r-1)$. Consider the set of vectors $z=\sum_{i=1}^{n} \mu_{i} z_{i}$, where $\mu_{i}$ are numbers such that $\|z\| \leq 1$. In Euclidean space with the norm generated by vectors $\left(\mu_{1}, \mu_{2}, \ldots, \mu_{n}\right)$ they form a convex body $B$ having the origin as a center. According to Lemma 2 there exist 
vectors $x_{1}, x_{2}, \ldots, x_{r}$ on the boundary of a set $B$ with the following property: for every numbers $\theta_{1}, \theta_{2}, \ldots, \theta_{r}$ with $\left|\theta_{i}\right| \leq 1,1 \leq i \leq r$,

$$
\sum_{i=1}^{r} \theta_{i} x_{i} \in \theta B, \text { where } \theta^{2}=3 \sum_{i=1}^{r} \theta_{i}^{2} \leq 3 r .
$$

Since for all $i=1,2, \ldots, r$ vectors $x_{i}$ belong to the boundary of $B$ they have unit norm in $X$. Since $\|z\| \leq 1$ for all $z \in B$ it follows from (1) that

$$
\left\|\sum_{i=1}^{r} \theta_{i} x_{i}\right\|^{2} \leq 3 r
$$

Now we can complete the proof of the necessity in Theorem 3. For simplicity we suppose $[a, b]=[0,1]$. Let $C$ be the Cantor ternary set, $\left(a_{i}^{r}, b_{i}^{r}\right)$, $r \geq 0,1 \leq i \leq 2^{r}$, being the intervals of rank $r$ contiguous to $C$ (we have $\left.b_{i}^{r}-a_{i}^{r}=3^{-r-1}\right)$ and $d_{i}^{r}$ being the middle points of the intervals $\left(a_{i}^{r}, b_{i}^{r}\right)$.

Assume that $X$ is of the infinite dimension. According to Lemma 3, for every $r$ we may construct vectors $x_{1}^{r}, x_{2}^{r}, \ldots, x_{2^{r}}^{r} \in X$ such that

$$
\left\|x_{i}^{r}\right\|=\frac{1}{2^{r}}, 1 \leq i \leq 2^{r},
$$

and for every numbers $\theta_{1}^{r}, \theta_{2}^{r}, \ldots, \theta_{2^{r}}^{r}$, with $\left|\theta_{i}^{r}\right| \leq 1,1 \leq i \leq 2^{r}$,

$$
\left\|\sum_{i=1}^{2^{r}} \theta_{i}^{r} x_{i}^{r}\right\|^{2} \leq \frac{3}{2^{r}}
$$

Define the function $f:[0,1] \longrightarrow X$ in the following way

$$
f(t)=\left\{\begin{array}{cc}
0, \text { if } t \in C \text { or } t=d_{i}^{r}, & r \geq 0,1 \leq i \leq 2^{r}, \\
2 \cdot 3^{r} x_{i}^{r}, \text { if } t \in\left(a_{i}^{r}, d_{i}^{r}\right), & r \geq 0,1 \leq i \leq 2^{r}, \\
-2 \cdot 3^{r} x_{i}^{r}, \text { if } t \in\left(d_{i}^{r}, b_{i}^{r}\right), & r \geq 0,1 \leq i \leq 2^{r} .
\end{array}\right.
$$

It is proved in [12] that this function is $M$-integrable (and consequently $H$ integrable) and its indefinite integral $F(\Delta)$ is not an $A C G^{*}$-function. Hence in view of the Proposition 2 function $f$ is not $D_{*} B$-integrable (and is not $B$ integrable) and therefore Theorems 1 and 2 imply that $f$ is not $V$-integrable and is not $M V$-integrable. This completes the proof. 


\section{References}

[1] Jr. S. R. Canoy and M. P. Navarro, A Denjoy-type integral for Banachvalued functions, Rend. Circ. Mat. Palermo 44 (1995), no. 2, 330-336.

[2] S. S. Cao, Banach-valued Henstock integration, Real Analysis Exchange 19 (1993-94), no. 1, 34.

[3] S. S. Cao, The Henstock integral for Banach-valued functions, SEA Bull. Math. 16 (1992), no. 1, 35-40.

[4] W. Congxin and Y. Xiaobo, A Riemann-type definition of the Bochner integral, J. Math. Study 27 (1994), no. 1, 32-36.

[5] A. Dvoretzky and C. A. Rogers, Absolute and unconditional convergence in normed linear spaces, Proc. Nat. Acad. Sci. USA 36 (1950), no. 3, 192-197.

[6] D. H. Fremlin, The Henstock and McShane integrals of vector-valued functions, Illinois J. Math. 38 (1994), no. 3, 471-479.

[7] R. Gordon, Equivalence of the generalized Riemann and restricted Denjoy integral, Real Analysis Exchange 12 (1986-87), no. 2, 551-574.

[8] R. Gordon, The Denjoy extension of the Bochner, Pettis and Dunford integrals, Studia Math. 92 (1989), 73-91.

[9] R. Gordon, The McShane integration of Banach-valued functions, Illinois J. Math. 34 (1990), 557-567.

[10] K. M. Ostaszewski, Henstock integral in the plane, Mem. of Amer. Math. Soc. 63(353) (1986), 1-106.

[11] S. Saks, Theory of the integral, Dover, New York, 1964.

[12] A. P. Solodov, Henstock and McShane integrals for Banach-valued functions, Mat. Zametki (to appear).

[13] A. P. Solodov, Riemann-type definition for the restricted Denjoy-Bochner integral, Fundamental and Applied Mathematics (to appear). 